\title{
Translocation and encapsulation of siRNA inside carbon nanotubes
}

\author{
Santosh Mogurampelly* and Prabal K. Maiti ${ }^{\dagger}$ \\ Centre for Condensed Matter Theory, Department of Physics, \\ Indian Institute of Science, Bangalore 560012, India
}

\begin{abstract}
We report spontaneous translocation of small interfering RNA (siRNA) inside carbon nanotubes (CNTs) of various diameters and chirality using all atom molecular dynamics (MD) simulations with explicit solvent. We use Umbrella sampling method to calculate the free energy landscape of the siRNA entry and translocation event. Free energy profiles shows that siRNA gains free energy while translocating inside CNT and barrier for siRNA exit from CNT ranges from 40 to $110 \mathrm{kcal} / \mathrm{mol}$ depending on CNT chirality and salt concentration. The translocation time $\tau$ decreases with the increase of CNT diameter with a critical diameter of $24 \AA$ for the translocation. In contrast, double strand DNA (dsDNA) of the same sequence does not translocate inside CNT due to large free energy barrier for the translocation. This study helps in understanding the nucleic acid transport through nanopores at microscopic level and may help designing carbon nanotube based sensor for siRNA.
\end{abstract}

${ }^{*}$ To whom correspondence should be addressed; Electronic address: santosh@ physics.iisc.ernet.in

†Electronic address: maiti@physics.iisc.ernet.in 


\section{INTRODUCTION}

Small interfering RNA (siRNA) is 21-23 nucleotides long double stranded RNA (dsRNA) which has been demonstrated to treat diseases like cancer and hepatitis. For such medical applications, siRNA has to be delivered inside the cell without degradation by complexing it with delivering vectors. However, efficient method for the delivery of siRNA to the target cell is lacking. Carbon nanotubes (CNTs) are cylindrical rolled graphene sheets which have emerged as new nano material to be used for biomedical applications due to their potential strengths [1-4]. CNTs have ultrahigh surface area which make them suitable drug delivery vectors through specific binding of biomolecules, drugs on their outer surface. This unique feature along with the other physical, thermal and optical properties enable high throughput drug delivery and medical imaging [2]. CNTs are not soluble in many solvents and also known to be toxic to the cell. This problem has been addressed by covalent or non-covalent surface functionalization of CNTs [1-4]. Delivery of RNA by translocating RNA-CNT hybrid through membranes of MCF7 breast cancer cells has also been studied [5]. siRNA delivery through pristine and functionalized CNT have been investigated by several groups in recent years. In this delivery approach siRNA is adsorbed on the CNT. After transfecting siRNA-CNT hybrid inside cells, the siRNA dissociates from the CNT. Recently we have shown that siRNA can adsorb onto the surface of the CNT and graphene by unzipping its base-pairs and binds strongly with high stability which can be used to deliver siRNA $[6,7]$. All these studies are concerned with siRNA adsorption/binding on CNT surface only. In this paper, we propose encapsulation of siRNA inside CNT as a possible delivery mechanisms.

Transport of biomolecules across cell membranes and nanopores is an important process in living organisms [8]. Single stranded DNA (ssDNA) transport in biologically occurred nanometer scale $\alpha$-hemolysin ion channel has been observed by Kasianowicz et. al. [9] in 1996. They observed that the translocation of ssDNA depends on the DNA concentration, applied voltage and structure of the nanopore. Since then, biomolecular transport in nanopores has become a major research activity both from fundamental and application point of view [10-12]. Polymer translocation through membranes [13] and nanopores [11, 14, 15] has been studied theoretically as a diffusion process across free energy barrier that arises due the chemical potential gradients. It has been found that the translocation time is proportional to the polymer length. Sophisticated 
nanopore based DNA sequencing techniques are now-a-days feasible to determine the sequence of DNA by measuring ionic currents as ssDNA translocates through the nanopore [16, 17]. However, microscopic level details are necessary for the better understanding of the DNA sequencing at the single base-pair level which is the ultimate goal of DNA sequencing technology. Translocation of ions, nucleic acids through solid state nanopores have been studied by Dekker and co-workers $[16,18,19]$. Translocation of oligonucleotides inside CNT have been reported as well [20-25]. Recently, translocation of DNA through nanopores in suspended graphene sheets have also been studied experimentally that finds applications in DNA sequencing [26-29].

Based on our MD simulation studies, we propose two different methods of siRNA delivery using CNTs and graphene. The first mechanism is based on the strong adsorption of siRNA on the CNT/graphene surface $[6,7]$ and the second one is by encapsulation of siRNA through translocation inside the CNT. The translocation of siRNA inside a CNT has not been studied earlier. In this paper, we report for the first time, the translocation studies of siRNA inside CNT. This translocation is driven by the favorable van der Waals ( $\mathrm{vdW}$ ) interaction between the siRNA and CNT. To understand the free energy landscape of siRNA translocation inside CNT, we have also calculated the free energy profile using Umbrella sampling method [30-32] for CNTs of different chirality and at different salt concentrations. The free energy profile indicates that siRNA gains energy inside CNT and faces a large energy barrier to escape from the interior of CNT. Insights into siRNA-CNT interaction and structural changes will help in understanding the microscopic picture of the siRNA delivery mechanism by CNTs. The translocation time $\tau$ of siRNA is decreasing with increasing the diameter of the CNT. Our simulation results can guide experimental approach for siRNA translocation inside CNT for sequencing and drug delivery applications as well. The rest of the of the paper is organized as follows: in the section II, we give the details of the simulation methods, followed by the discussion on the results of spontaneous translocation. In section III, we discuss the results on free energy landscapes using MM-GBSA as well as Umbrella sampling method. Finally in section IV, we give a summary of the main results and conclude.

\section{METHODS}

We have built single walled CNT (SWCNT) of different chiralities and diameters of length approximately $87 \AA$. The diameters $d$ of the built SWCNTs are $27.01 \AA, 26.74 \AA, 25.40 \AA, 24.06$ 
$\AA$ and $22.73 \AA$ for $(25,15),(20,20),(19,19),(18,18)$ and $(17,17)$ nanotubes respectively. We study several systems for nucleic acid translocation inside CNTs: (i) siRNA translocation mechanism inside $(20,20) \mathrm{CNT}$ at 0 and $107 \mathrm{mM} \mathrm{NaCl}$ concentration to investigate the effect of salt concentration (ii) siRNA translocation inside $(19,19),(18,18)$ and $(17,17)$ CNTs to study the effect of diameter (iii) siRNA translocation mechanism inside $(25,15)$ to study the effect of chirality and finally (v) dsDNA (of identical sequence except adenine instead of uracil of siRNA) translocation mechanism inside $(20,20) \mathrm{CNT}$ to understand the difference between the interaction of siRNA and dsDNA with CNT. For all these cases, the siRNA/dsDNA was placed close to one of the open ends of CNT such that the CNT axis and the siRNA axis are parallel. We have used the same siRNA sequence and structure as described in our earlier publication $[6,7]$. We have used ff99 force field [33] for all the simulations reported in this paper. The siRNA-CNT system is solvated with water and counterions. To make a charge neutral system for different salt concentration, appropriate number of $\mathrm{Na}^{+}$and $\mathrm{Cl}^{-}$ions were added. The system was then subjected to standard simulation protocol $[34,35]$ with periodic boundary conditions applied in all three directions. Non-bonded interactions are truncated at $9 \AA$ and the long range electrostatic interactions were calculated with the Particle Mesh Ewald (PME) method [36]. Finally for trajectory analysis, simulations are performed in constant volume-constant temperature (NVT) ensemble up to 100 ns depending on the diameter of CNT. For the Umbrella sampling method, 120-130 ns of NVT simulations are performed for each system. Full system details such as number of $\mathrm{NaCl}$ ions, water residues, box dimensions and total number of atoms are summarized in Table I. To check the

effect of force field, we have also performed the simulation with $(20,20)$ CNT and siRNA using ff10 force field [37].

\section{RESULTS AND DISCUSSION}

\section{A. Translocation of siRNA inside CNTs}

Snapshots shown in Figure 1 represent the translocation process of siRNA inside (20, 20) CNT at various instants of time. To present the details of the system studied, we show the initial siRNA$(20,20)$ CNT system with counterions and water in Figure 1(a). In Figures 1(b) to 1(i), we show the instantaneous snapshots of the system in few ns interval. For clarity, water and counterions are not shown. From the snapshots and subsequent analysis, we observe that the siRNA translocates 
into the interior of $(20,20)$ CNT from the initial configuration. The translocation of siRNA inside $(20,20) \mathrm{CNT}$ is very fast and happens in $17.4 \mathrm{~ns}$. After the translocation, siRNA stays inside CNT with least positional fluctuations during the remaining simulation period of 30-40 ns. We have also observed the translocation of siRNA at $107 \mathrm{mM}$, inside $(19,19),(18,18)$ and $(25,15)$ CNTs. The siRNA inside $(20,20) \mathrm{CNT}$ at $0 \mathrm{mM}$ has least structural deformations compared to all other CNTs of different diameter and chirality studied in this work. Similar results are observed at $107 \mathrm{mM} \mathrm{NaCl}$ concentration also. In all these cases, the siRNA is very stable inside CNT without any further movement after the translocation. This siRNA-CNT hybrid can serve as siRNA delivery vector for the delivery of siRNA for RNAi therapy. For the siRNA release after delivery, functionalizing siRNA with nanoparticles or application of electric field can be used.

To understand the energetics of siRNA as it translocates inside $(20,20) \mathrm{CNT}$, we have calculated the van der Waals (vdW) interaction energy $\phi(r)$ between siRNA and CNT at various instants of time. We plot $\phi(r)$ as a function of the siRNA spatial position $r$ at various instants of time $t$ in Figure 2. Figure 2 shows how different parts of siRNA interact with CNT and also the interaction strength along CNT axis $\hat{n}$. In Figure 2, $r=0$ corresponds to the center of mass (COM) position of siRNA. For the calculation of $\phi(r)$, siRNA has been divided into 4 parts along its helix axis. The data shown in Figure 2 represents how the COM of these 4 parts of siRNA interact with CNT as it translocates. It can be seen from the Figure 2 that $\phi(r)$ has different shapes at $t=0 \mathrm{~ns}, 10$ ns, $15 \mathrm{~ns}, 25 \mathrm{~ns}$ and $40 \mathrm{~ns}$. Initially siRNA is outside CNT with only two sticky-ends (these are un-paired nucleobases in a nucleic acid) and one intact Watson-Crick (WC) base-pair lying inside CNT. Hence the interaction of siRNA with CNT is very less which are far apart from each other (see $t=0$ ns curve in Figure 2). As $t$ increases, these sticky-ends interact strongly with CNT as shown in Figure 2 at $t=10 \mathrm{~ns}, 15 \mathrm{~ns}, 25 \mathrm{~ns}$ and $40 \mathrm{~ns}$. The siRNA strongly interacts with CNT after complete translocation for $t \geq \tau$; where $\tau$ is the translocation time. The increase of $\phi$ after complete translocation is about $407 \mathrm{kcal} / \mathrm{mol}$ or $690.8 k_{B} T$. 


\section{B. Free energy landscape during translocation}

\section{Free energy using equilibrium simulation: MM-GBSA and 2PT method}

In general, the binding free energy for the non-covalent association of two molecules $\mathrm{A}$ and B may be written as $\Delta G(A+B \rightarrow A B)=G_{A B}-G_{A}-G_{B}$. For any species on the right hand side $G(X)=H(X)-T S(X)$, where $H$ is the enthalpy, $T$ is the absolute temperature and $S$ is the entropy of the molecule. Therefore the binding energy at constant temperature can be written as

$$
\Delta G=\Delta H-T \Delta S
$$

The calculation of enthalpy difference $(\Delta H)$ and entropy difference $(\Delta S)$ contributions to the binding free energy in Eqn. 1 are done using molecular mechanics - generalized Born surface area (MM-GBSA) method and 2 phase thermodynamic (2PT) method [38-40], respectively. The change in enthalpy $\Delta H$ can be decomposed into the gas-phase energy $\left(\Delta E_{\mathrm{gas}}\right)$ and solvation free energy $\left(\Delta G_{\text {sol }}\right)$; i.e., $\Delta H=\Delta E_{\text {gas }}+\Delta G_{\text {sol }}$. For any species $A, B$ or complex $A B$, the gas-phase energy, $E_{\mathrm{gas}}$ is calculated from molecular mechanics using $E_{\mathrm{gas}}=E_{\mathrm{ele}}+E_{\mathrm{vdw}}+E_{\mathrm{int}}$, where, $E_{\text {ele }}$ is the electrostatic energy calculated from the Coulomb potential, $E_{\mathrm{vdw}}$ is the non-bonded van der Waals energy and $E_{\text {int }}$ is the internal energy contribution arising from bond stretching, angle bending and dihedrals. On the other hand, the solvation free energy, $G_{\text {sol }}$ can be further decomposed as electrostatic $G_{\mathrm{el}}$ and non-electrostatic $G_{\text {non-el }}$ contributions; i.e., $G_{\mathrm{sol}}=G_{\mathrm{el}}+G_{\text {non-el }}$. The electrostatic energy, $G_{\mathrm{el}}$ is calculated from Generalized Born (GB) method which assumes that the atoms in a molecule are spheres of radius $R_{i}$ (called Born radius) and have an effective charge $q_{i}$ (called Born charge). The molecule is assumed to be surrounded by a solvent of dielectric constant $\varepsilon$ ( 80 for water at $300 \mathrm{~K}$ ) and the solute atoms have a dielectric constant of 1 . The analytic expression for the $G_{\text {el }}$ in the GB model $[41,42]$ is given by

$$
G_{\mathrm{el}}=-\frac{1}{2} \sum_{i, j} \frac{q_{i} q_{j}}{f_{G B}\left(r_{i j}, R_{i}, R_{j}\right)}\left(1-\frac{\exp \left(-\kappa f_{G B}\right)}{\varepsilon}\right)
$$

where

$$
f_{G B}\left(r_{i j}, R_{i}, R_{j}\right)=\left[r_{i j}^{2}+R_{i} R_{j} \exp \left(-r_{i j}^{2} / 4 R_{i} R_{j}\right)\right]^{\frac{1}{2}}
$$

where $r_{i j}$ is the distance between atoms $i, j$ and $R_{i}$ are effective Born radii and $\mathrm{\kappa}$ is the DebyeHückel screening parameter. The non-electrostatic energy, $G_{\text {non-el }}$ is calculated as $\gamma \times S A S A+\beta$; 
where $\gamma$ is the surface tension parameter $\left(\gamma=0.0072 \mathrm{kcal} / \mathrm{mol}-\AA^{2} ; \beta=0 \mathrm{kcal} / \mathrm{mol}\right)$ and $S A S A$ is the solvent-accessible surface area of the molecule. The entropy contribution appearing in Eqn. 1 is carried out using 2 phase thermodynamic approach proposed by Lin et. al. [38-40] which is motivated by the observation that the density of states $(S(v))$ of a liquid can be decomposed into a gas component and a solid component [38-40]. The density of states function can be calculated from the Fourier transform of velocity auto-correlation function; i.e.,

$$
S(v)=\frac{2}{k_{B} T} \lim _{\tau \rightarrow \infty} \int_{-\tau}^{\tau} \sum_{j=1}^{N} \sum_{k=1}^{3} m_{j} c_{j}{ }^{k}(t) e^{-i 2 \pi v t} d t
$$

where $c_{j}^{k}(t)$ is the velocity auto-correlation function which is given by

$$
c_{j}{ }^{k}(t)=\lim _{\tau \rightarrow \infty} \frac{1}{2 \tau} \int_{-\tau}^{\tau} v_{j}^{k}(t) v_{j}^{k}\left(t+t^{\prime}\right) d t^{\prime}
$$

The entropy $S$ is then calculated from the knowledge of $S(v)$ using

$$
S=k_{B} \ln Q+\beta^{-1} \frac{\partial \ln Q}{\partial T}_{N, V}
$$

where the partition function $Q$ is given by

$$
\ln Q=\int_{0}^{\infty} d v S(v) \ln q_{H O}(v)
$$

The decomposition of $S(v)$ into gas-phase and solid-phase is performed to get the entropy of molecular species $A, B$ or complex $A B$ to get finally the change in free energy $T \Delta S$. The $2 \mathrm{PT}$ method has found successful application in several related problems [38-40, 43-45].

The binding free energy $(\Delta G)$ as function of time is shown in Figure 3(a). Entropy $(T \Delta S)$ and enthalpy $(\Delta H)$ contributions to $\Delta G$ are shown in the inset. $\Delta S$ decreases with time since the fluctuations in siRNA nucleobases are suppressed by CNT making less microstates available for siRNA as translocation progresses. However, $\Delta H$ is increasing with time and attains constant value after complete translocation. $\Delta G$ also follows similar trend since the entropy contribution is very small compared to enthalpy contribution. We plot the vdW contribution $\Delta \phi$ to the total binding energy in Figure 3(b). When siRNA and CNT are far apart in the initial stage, $\Delta \phi$ is very less but increases as siRNA translocates inside CNT. We also show instantaneous snapshots of the siRNA-CNT hybrid at various instants of time $t$ in Figure 3(b). $\Delta \phi$ is $-900 \mathrm{kcal} / \mathrm{mol}$ after complete translocation of siRNA compared to $-85 \mathrm{kcal} / \mathrm{mol}$ at the initial stage. The interaction between 
siRNA and CNT is driven mainly by vdW interaction (Figure 3(b)). This is similar to our earlier results that the unzipping and adsorption of siRNA on CNT/graphene is also driven by the $\mathrm{vdW}$ interaction [6,7]. The conversion of time into distance between CNT and siRNA while plotting $\Delta G(r)$ has been discussed in the supplementary materials [46].

\section{Free energy using Umbrella sampling method}

In the un-biased MD simulations discussed so far, siRNA has translational motion in 1-D along nanotube axis $\hat{n}$ in order to translocate into the interior of CNT. The free energy landscape of the siRNA translocation process along such suitable reaction coordinate will help in understanding the stable and unstable states for siRNA with respect to the CNT. In the previous section we have described the free energy calculation from the equilibrium un-biased MD simulation using a combination of MM-GBSA and 2PT methods. Such methods in equilibrium MD cannot properly account for the sampling of high energy states. For this reason, we use Umbrella sampling method [30] to sample the entire phase space along chosen reaction coordinate and calculate the free energy profile for siRNA translocation inside CNT. The basic idea implemented in constructing the free energy is to add a biasing potential to obtain sampling of less probable states for siRNA translocation process and record the biased histograms. This is done for a series of biasing potentials at various values of chosen reaction coordinate that span the entire translocation path of interest. We make sure that the successive histograms have enough overlap in order to reconstruct the un-biased free energy from biased histograms. Below we give a brief overview of the Umbrella sampling method. For further details, readers are refereed to the excellent text book by Frenkel and Smit [32].

With the unperturbed potential $V\left(\mathbf{r}^{N}\right)$, we have added a biasing harmonic potential $U_{i}(\xi)=$ $\frac{1}{2} k\left(\xi-\xi_{i}\right)^{2}$, resulting in the perturbed potential $V^{\prime}\left(\mathbf{r}^{N}\right)=V\left(\mathbf{r}^{N}\right)+\sum_{i=1}^{N_{W}} U_{i}(\xi)$; where $\xi_{i}$ is the restrained distance between the center of masses of 'far end' of CNT and first two Watson-Crick hydrogen bonded base-pairs of siRNA close to the CNT for $i^{\text {th }}$ window. The values of $k$ and $\Delta \xi$ should be chosen optimally such that the phase space is properly sampled within reasonable time scales. Therefore, we have optimized the force constant $k$ of $U_{i}(\xi), \Delta \xi$ and equilibration time before performing the Umbrella sampling. The optimized values are $k=4 \mathrm{kcal} / \mathrm{mol}-\AA^{2}, \Delta \xi_{i}=1$ $\AA$ and equilibration time of $1 \mathrm{~ns}$ to ensure proper sampling and overlapping of successive his- 
tograms, $P_{i}^{\prime}(\xi)$. The values of $\xi_{i}$ is changed from $130 \AA$ to $10 \AA$ until the complete translocation happens in steps of $1 \AA$ totaling $N_{w}=120$ Umbrella simulations (windows) of each 1 nano second duration. The probability distribution of the reaction coordinate $\xi$ separating CNT and siRNA is,

$$
\begin{aligned}
P_{i}(\xi) & =\left\langle\delta\left(\xi-\xi_{i}\right)\right\rangle \\
& =\frac{1}{Z} \int d \mathbf{r}^{N} \delta\left(\xi-\xi_{i}\right) \exp \left[-\beta V\left(\mathbf{r}^{N}\right)\right]
\end{aligned}
$$

Similarly the probability distribution in the presence of $U_{i}(\xi)$ is

$$
\begin{aligned}
P_{i}^{\prime}(\xi) & =\frac{1}{Z^{\prime}} \int d \mathbf{r}^{N} \delta\left(\xi-\xi_{i}\right) e^{-\beta\left[V\left(\mathbf{r}^{N}\right)+U_{i}(\xi)\right]} \\
& =\frac{Z}{Z^{\prime}} e^{-\beta U_{i}(\xi)} P_{i}(\xi) .
\end{aligned}
$$

Re-arranging this gives,

$$
P_{i}(\xi)=\frac{Z^{\prime}}{Z} e^{\beta U_{i}(\xi)} P_{i}^{\prime}(\xi)
$$

here, $\delta$ is a Dirac delta function, $N$ is the number of atoms, $\mathbf{r}^{N}$ denotes the set of atom coordinates, $\beta=1 / k_{B} T$, where $k_{B}$ is the Boltzmann constant, $T$ is the absolute temperature, $V\left(\mathbf{r}^{N}\right)$ is the potential energy and $Z, Z^{\prime}$ are the partition functions of un-biased and biased systems, respectively. Here problem arises due to the fact that $P(\xi)$ becomes exceedingly small for values of $\xi$ which give significant contribution to the free energy. Umbrella sampling makes use of a biasing potential to sample the region of phase space for which $P(\xi)$ is exceedingly small. Thus, the distribution function $P(\xi)$ can be obtained using Eqn. 8 (to within a multiplicative constant) from the measurement of the biased distribution $P^{\prime}(\xi)$. The un-biased free energy $(F)$ was constructed self-consistently using the weighted histogram analysis method (WHAM) [30-32] with the following Eqns. 9 and 10.

where $F_{i}$ is given by

$$
P(\xi)=\frac{\sum_{i=1}^{N_{w}} n_{i} P_{i}(\xi)}{\sum_{j=1}^{N_{w}} n_{j} e^{-\beta\left(U_{j}(\xi)-F_{j}\right)}}
$$

$$
e^{-\beta F_{i}}=\int d \xi e^{-\beta U_{i}(\xi)} P(\xi)
$$

where $n_{i}$ is the number of data points in $i^{\text {th }}$ window. By piecing together the relative free energies measured using a number of biasing potentials, we construct $F(\xi)$ over the chosen range of $\xi=$ $10 \AA$ to $130 \AA \AA$.

Figure 4(a) shows the free energy $F(\xi)$ for siRNA in $(20,20) \mathrm{CNT}$ at $0 \mathrm{mM} \mathrm{NaCl}$ concentration. We find that when siRNA is outside $(20,20) \mathrm{CNT}$, the free energy is zero since there is no 
interaction between siRNA and $(20,20)$ CNT. The snapshots at various time instants show the translocation process as $\xi$ is decreasing from $130 \AA$ to $10 \AA$. For $\xi \geq 105 \AA$, siRNA is outside $(20,20) \mathrm{CNT}$ and does not interact with it. As the value of $\xi$ is decreased from $105 \AA, F(\xi)$ decreases to minima at $92 \AA$ at which siRNA is in a local stable state. At this stage siRNA rotates inside CNT and two unpaired bases of siRNA and one WC base-pair unzips to interact with (20, 20) CNT as can be seen in Figure 4(a). Further decrease in $\xi$ results in increasing $F(\xi)$ having an energy barrier for siRNA to translocate inside CNT. $F(\xi)$ reaches maximum at $\xi=80 \AA$ with an energy barrier of $\Delta G=9 \mathrm{kcal} / \mathrm{mol}$. The energy barrier for further translocation arises due to strong vdW interaction between unpaired nucleobases of siRNA and CNT. Thermal fluctuations help siRNA to overcome this barrier. Note that, once it overcomes this barrier, favorable vdW interaction between nucleobases and CNT helps siRNA to translocate inside. From $\xi=80 \AA$, siRNA goes inside $(20,20)$ CNT easily and $F(\xi)$ decreases until complete siRNA translocation at $\xi_{\min }=42 \AA$. This is the most stable position for siRNA inside $(20,20)$ CNT where siRNA stays for the rest of the simulation time.

Figure 4(b) presents free energy for siRNA in CNTs for $(20,20),(25,15)$ chiralities at 0 $\mathrm{mM} \mathrm{NaCl}$ concentration, for $(20,20) \mathrm{CNT}$ at $107 \mathrm{mM} \mathrm{NaCl}$ concentration and for dsDNA in $(20,20) \mathrm{CNT}$ at $0 \mathrm{mM} \mathrm{NaCl}$ concentration. In the un-biased simulation, we have observed the translocation of siRNA in CNTs but no translocation of dsDNA is observed. The dsDNA has favorable state only outside the $(20,20)$ CNT as can be seen in Figure 4(b). This might be due to the less favorable interaction of thymidine nucleobase with nanotube compared to the uridine nucleobase interaction with nanotube $[6,7]$. For $\xi \leq L / 2$, where $L$ is the length of the CNT, $F(\xi)$ is constantly increasing which means dsDNA encounters unfavorable free energy landscape when translocated inside $(20,20)$ CNT. This dramatic difference of interaction between CNT and siRNA and CNT and dsDNA may be due to the relatively weaker interaction strength of thymidine with CNT than that of uridine with CNT $[6,7]$. Moreover, the stronger WC base-pairing interaction energy of A-T compared to A-U $[6,7,47,48]$ makes dsDNA difficult to get unzipped. Earlier we have shown that unzipped base-pairs facilitate binding with CNT/graphene [6, 7]. dsDNA requires more than $100 \mathrm{kcal} / \mathrm{mol}$ energy to overcome a free energy barrier to translocate inside $(20,20)$ CNT which is not possible without any external force. However, possibility of the translocation of dsDNA in CNT of large diameter has not been studied in our simulation. In experiments of dsDNA translocation inside CNT, the diameter of CNT is $50-100 \mathrm{~nm}[21,49]$. In the experiments 
of ssDNA translocation through $\alpha$-hemolysin ion channel [9], the diameter of $\alpha$-hemolysin is 26 $\AA$ and the polymer is single stranded. Diameter of $(20,20)$ CNT is $26.74 \AA$ which is close to the diameter of $\alpha$-hemolysin ion channel and so the channel diameter is not sufficient for dsDNA translocation. We have observed the translocation of ssDNA through CNT of diameter 26.74 $\AA$ [50]. Our results on ssDNA and dsDNA translocation are consistent with experiments [9]. $F(\xi)$ for dsDNA has global minimum only outside the $(20,20)$ CNT. When dsDNA is forced to translocate inside $(20,20) \mathrm{CNT}$ with an external Umbrella potential, dsDNA deforms largely by breaking most of the WC base-pairs due to the large external force by Umbrella potential. To have further confirmation that siRNA translocate inside CNT and dsDNA does not, we have performed two separate simulations of siRNA and dsDNA by keeping them initially inside the $(20,20)$ CNT. As expected, siRNA stays inside the CNT for long time where as dsDNA comes out of the CNT within 500 ps. These results confirm that siRNA spontaneously translocates inside a $(20,20) \mathrm{CNT}$ without any external force where as dsDNA requires an external force in order to be translocated inside $(20,20)$ CNT.

To test the effect of chirality as well as salt concentration on the translocation event, we have calculated the free energy profile of siRNA translocation inside $(25,15) \mathrm{CNT}$ at $0 \mathrm{mM} \mathrm{NaCl}$ concentration and for $(20,20) \mathrm{CNT}$ at $107 \mathrm{mM} \mathrm{NaCl}$ concentration using Umbrella sampling method. The free energy profile for these cases are shown in Figure 4(b). In both these cases, the free energy minima is lower compared to the case of siRNA in $(20,20) \mathrm{CNT}$ at $0 \mathrm{mM}$ concentration of $\mathrm{NaCl}$. So for these cases we expect translocation to happen faster compared to the case for siRNA in $(20,20) \mathrm{CNT}$ at $0 \mathrm{mM} \mathrm{NaCl}$ concentration. Translocation time for various cases has been discussed in section III C. The inset of Figure 4(b) shows the minima of $F(\xi)$. We observe

that $\xi_{\min }=37 \AA$ at $107 \mathrm{mM}$ as compared to $\xi_{\min }=42 \AA$ at $0 \mathrm{mM}$. However, $\xi_{\min }=21 \AA$ for siRNA in $(25,15) \mathrm{CNT}$. The origin for this observation is discussed in the next section by analyzing un-biased simulations.

\section{Translocation time $(\tau)$}

The translocation of siRNA strongly depends on the diameter $d$ of CNT since the vdW interaction between siRNA and CNT increases as $d$ is decreased (nearly comparable to siRNA diameter). The translocation time, $\tau$ is defined as the time required for at least half of the siRNA base-pairs 
to enter inside the CNT; i.e., $r_{\text {com }} \leq L / 2$ which corresponds to $r_{\text {com }} \leq 43.5 \AA$ or $\xi \leq 59 \AA$, where $L=87 \AA$ is the length of the CNT. We plot $r_{\text {com }}$ and $\tau$ in Figures 5(a) and 5(b), respectively for various diameters of CNT. In Figure 5(a), we see that $r_{\text {com }}$ is decreasing with time as siRNA moves towards the interior of the CNT. However, the sticky-ends and unzipped nucleobases of siRNA interact with CNT in the course of translocation, and these give rise to very rugged free energy profile of siRNA during the translocation. We have calculated $\tau$ by tracking $r_{\text {com }}$ as a function of time as shown in Figure 5(a). The dashed horizontal curve drawn at $L / 2$ serves as the reference for translocation criteria. We find that $\tau$ is linearly decreasing with CNT diameter $d$. Similar dependence was observed for DNA transport through graphene nanopore [26] where the ionic conductance was found to be proportional to the pore diameter. We note that our results on $\tau$ are very sensitive to the initial model building. To see the effect of initial relative positioning of siRNA with respect to CNT, we simulated the translocation process for three different initial conditions for $(20,20) \mathrm{CNT}$. We find $\tau$ to be $18 \mathrm{~ns}, 14 \mathrm{~ns}$ and $17.4 \mathrm{~ns}$ and see a strong dependence of $\tau$ on the initial conditions. Hence our results on $\tau$ can only serve as qualitative understanding of the systems under investigation in this study. To understand the origin of this diameter dependence, we have calculated the number of contacts $N_{c}$ between the siRNA and CNTs and have shown them in Figure 5(c). $N_{c}$ of siRNA is calculated within $5 \AA$ from the inner surface of CNT that mostly represents the effective vdW interaction range. As shown in Figure 5(c), $N_{c}$ of siRNA are 110 at $t$ $=0 \mathrm{~ns}$ and rapidly increases to a constant value after complete translocation. The maximum value of $N_{c}$ increases with $d$ ranging from 520 inside the $(18,18)$ CNT to 815 inside the $(25,15)$ CNT. siRNA translocation happens above a critical CNT diameter of $24.0 \AA$ which corresponds to (18, 18) CNT, below which no translocation is observed. For siRNA in $(17,17) \mathrm{CNT}$ and dsDNA in $(20,20) \mathrm{CNT}$, the value of $N_{c}$ is constant and very less indicating no translocation in the simulated time scale. Since we have not observed the translocation of siRNA in $(17,17)$ CNT and dsDNA in $(20,20) \mathrm{CNT}, \tau$ is assumed to be very large. For the translocation of siRNA inside CNT, the minimum diameter of CNT, $d_{\min }$ should be greater than or equal to the diameter of siRNA + effective vdW radius of CNT and siRNA; i.e., $d_{\min } \geq d_{\mathrm{siRNA}}+d_{\mathrm{vdW}}$. The diameter of $(17,17) \mathrm{CNT}$ is 22.73 $\AA$ which is very close to siRNA diameter and hence cannot accommodate siRNA unless siRNA is severely stretched. As expected, with decreasing CNT diameter, the deformation in siRNA is more due to strong vdW interaction between siRNA and CNT. The structural aspects of siRNA during translocation are discussed in section III D. Interestingly, when the salt concentration is increased to $107 \mathrm{mM}$, $\tau$ decreases drastically to $4 \mathrm{~ns}$ compared to $17.4 \mathrm{~ns}$ at $0 \mathrm{mM}$ salt concentration. High 
salt provides better counterion condensation around phosphate atoms in siRNA backbone compared to $0 \mathrm{mM}$ case. The electrostatic screening increases as salt is increased from 0 to $107 \mathrm{mM}$ which reduces phosphate-phosphate electrostatic repulsion in the backbone of siRNA. Therefore, the stability to siRNA increases with increasing salt concentration which reduces the propensity of siRNA unzipping [6]. As a result, at $107 \mathrm{mM} \mathrm{NaCl}$ concentration, there is less binding of the siRNA with the CNT. So the translocation is faster at $107 \mathrm{mM}$ salt concentration and hence $\tau$ is small. At $107 \mathrm{mM}$ of $\mathrm{NaCl}$ concentration, the maximum value of $N_{c}$ is 730 which is 20 more in number than that of at $0 \mathrm{mM}$.

\section{Structural aspects of siRNA during translocation inside CNT}

In order to translocate, siRNA has to cross the free energy barrier arising due to loss of entropy of siRNA. In this process, many structural deformations occurs in siRNA. We have calculated several quantities that quantify the degree of structural deformations in siRNA. In Figure 6(a), we we plot the root mean square deviation (RMSd) as a function of time as siRNA translocates in the interior of CNT for various CNT diameters as well as for different salt concentrations. The RMSd was calculated with respect to the initial minimized structure of siRNA. The RMSd of the siRNA which is inside of a thinner CNT is larger than that of the siRNA which is inside a fatter CNT. This difference is due to the large structural deformation of siRNA inside CNT of smaller diameter. The average RMSd of siRNA is $3.0 \AA, 5.4 \AA$ and $8.2 \AA$ inside $(20,20),(19,19)$ and $(18,18)$ CNT, respectively. Also RMSd for siRNA in $(20$, 20) $\mathrm{CNT}$ is higher at $107 \mathrm{mM}$ salt concentration compared to $0 \mathrm{mM}$ concentration. Among all the cases, siRNA in $(20,20) \mathrm{CNT}$ at $0 \mathrm{mM}$ has least RMSd after translocation with small fluctuations. In the delivery application, the translocation method is more appropriate and diameter of the CNT can be appropriately chosen to have minimum deformation of siRNA structure.

To translocate inside smaller diameter CNTs, siRNA has to get unzipped. To quantify the amount of unzipping of siRNA inside CNT, we have calculated the number of WC H-bonds based on geometry criteria. WC H-bonds play a crucial role in maintaining the double stranded form of nucleic acids and their function [51-53]. For WC H-bond calculation, we have used geometry based criteria wherein for H-bond, donor-acceptor (D-A) distance $\leq 2.7 \AA$ and angle $\angle \mathrm{DHA} \geq 130^{\circ}$. We have plotted the WC H-bonds of siRNA in various CNTs in Figure 6(b). 
There are only three broken H-bonds out of total 48 possible H-bonds for siRNA in the case of $(20,20) \mathrm{CNT}$ at 0 and $107 \mathrm{mM}$ salt concentrations and dsDNA in the case of $(20,20) \mathrm{CNT}$ at 0 $\mathrm{mM}$ salt concentration. As $d$ is decreasing, more H-bonds are broken in siRNA due to stronger $\mathrm{vdW}$ interaction between siRNA and CNT. Interestingly, large number of H-bonds of siRNA are broken inside $(25,15) \mathrm{CNT}$. The aromatic rings of $(25,15) \mathrm{CNT}$ are oriented differently with respect to $\mathrm{CNT}$ axis $\hat{n}$. Hence nucleobases try to orient along the aromatic ring direction due to $\mathrm{vdW}$ interaction resulting in large number of broken $\mathrm{H}$-bonds. The larger number of broken H-bonds for smaller diameter is also reflected in the larger RMSd of siRNA in case of smaller diameter as shown in Figure 6(a).

Another very interesting observation is the rotation of siRNA to optimize the nucleobase orientation with respect to the aromatic rings of CNT while translocating inside CNT. We have calculated the distribution of rotation angle, $P(\theta)$ of siRNA where $\theta$ is the average rotation angle of siRNA as it translocates inside nanotube with respect to the structure outside nanotube. The rotation angle $\theta$ is calculated using

$$
\theta(t)=\left\langle\cos ^{-1}\left(\mathbf{p}_{i}(t) \cdot \mathbf{p}_{i}(0)\right)\right\rangle_{i}
$$

where $\mathbf{p}_{i}(t)$ is the vector joining phosphate-phosphate atoms of $i^{t h}$ base-pair for $i=4,5 \ldots 17$ at time $t$. The angular brackets $\langle\ldots\rangle_{i}$ denote average over $i$ base-pairs. Since sticky-ends and basepairs near to both the ends of siRNA have large fluctuations compared to the middle part of siRNA, they are omitted for the calculation of $\theta . P(\theta)$ for siRNA while translocation inside various CNTs is plotted in Figure 6(c). We find that siRNA has to rotate in order to translocate inside CNT. Several mechanisms of DNA packaging propose the rotation of DNA during translocation [5456]. By rotating, siRNA get reoriented with respect to the CNT inner surface which helps in overcoming local free energy barriers and helps in the translocation. Rotation is large for siRNA inside $(20,20) \mathrm{CNT}$ at $0 \mathrm{mM}$ salt concentration and is least for siRNA inside $(18,18) \mathrm{CNT}$ with $P(\theta)$ having very strong peak at $132^{\circ}$ and $35^{\circ}$, respectively. In the case of other CNTs, $P(\theta)$ has more than one peak with comparable magnitudes indicating that those rotations are equally likely to be observed. Hence, the local free energy barriers are escaped by rotation of siRNA in order to translocate inside CNTs. 


\section{CONCLUSION}

To conclude, all atom molecular dynamics simulations with explicit solvent are used to investigate the translocation and encapsulation of siRNA inside CNTs of various diameters, chiralities at various salt concentrations. After the translocation, the siRNA stays inside without any further movement. Free energy calculation using Umbrella sampling shows that siRNA gains in free energy while translocating inside CNT and has to overcome large free energy barrier to exit from the interior of the nanotube. There is no translocation of siRNA inside $(17,17)$ CNT which has a diameter of $22.73 \AA$. We find that the diameter of the $(18,18) \mathrm{CNT}$ is the critical diameter for the translocation. Interestingly, siRNA translocates inside $(20,20)$ CNT but dsDNA cannot. This difference is due to (i) more interaction strength of uridine with CNT inner surface than that of thymidine [6,7] and (ii) strong A-T base-pairing energy [6, 7, 47, 48] compared to A-U basepairing energy. The translocation time $\tau$ is decreasing with increasing diameter of the CNT with a critical diameter of $24 \AA$. Inside CNTs of smaller diameters, vdW repulsion is larger which causes more structural deformations in siRNA. Free energy barrier for exit as well as translocation time $\tau$ strongly depends on the chirality of the nanotube. The aromatic ring orientation in $(25,15) \mathrm{CNT}$ also induces large structural deformations in siRNA and makes the siRNA and CNT interaction stronger than the $(20,20)$ CNT having same diameter. This makes the translocation in $(25,15)$ CNT faster compared to $(20,20)$ CNT. Among all the systems studied, siRNA has least deformations when translocated inside $(20,20)$ CNT. The stable siRNA-CNT hybrid can be used to deliver siRNA in RNAi applications. The kinetics and other thermodynamic analysis presented in this work allow us to get a microscopic understanding of the translocation process. By means of rotation, siRNA escapes from the local free energy barriers and translocate inside nanotubes. Modeling CNT with partial atomic charges on carbon atoms, functionalizing siRNA with polar nanoparticles for the efficient drug delivery systems are among exciting future perspectives in this emerging area. 


\section{ACKNOWLEDGEMENTS}

We thank department of biotechnology (DBT), India for the financial support.

[1] Z. Liu, M. Winters, M. Holodniy, and H. Dai. Angew. Chem. Int. Ed., 46(12):2023-2027, (2007).

[2] Z. Liu, S. Tabakman, K. Welsher, and H. Dai. Nano Research, 2(2):85-120, (2009).

[3] N. Kam, Z. Liu, and H. Dai. Angew. Chem. Int. Ed., 45(4):577-581, (2006).

[4] Z. Zhang, X. Yang, Y. Zhang, B. Zeng, Z. Wang, T. Zhu, R. B. S. Roden, Y. Chen, and R. Yang. Clin. Cancer. Res., 12(16):4933-4939, (2006).

[5] Q. Lu, J. Moore, G. Huang, A. Mount, A. Rao, L. Larcom, and P. Ke. Nano Lett., 4(12):2473-2477, (2004).

[6] M. Santosh, S. Panigrahi, D. Bhattacharyya, A. K. Sood, and P. K. Maiti. J. Chem. Phys., 136(24):065106, (2012).

[7] S. Mogurampelly, S. Panigrahi, D. Bhattacharyya, A. K. Sood, and P. K. Maiti. J. Chem. Phys., 137(5):054903, (2012).

[8] H. Kumar, Y. Lansac, M. A. Glaser, and P. K. Maiti. Soft Matter, 7(13):5898-5907, (2011).

[9] J. Kasianowicz, E. Brandin, D. Branton, and D. Deamer. Proc. Natl. Acad. Sci. USA., 93(24):1377013773, (1996).

[10] S. Henrickson, M. Misakian, B. Robertson, and J. Kasianowicz. Phys. Rev. Lett., 85(14):3057-3060, (2000).

[11] D. Lubensky and D. Nelson. Biophys. J., 77(4):1824-1838, (1999).

[12] A. Meller, L. Nivon, and D. Branton. Phys. Rev. Lett., 86(15):3435-3438, (2001).

[13] W. Sung and P. Park. Phys. Rev. Lett., 77(4):783-786, (1996).

[14] M. Muthukumar. J. Chem. Phys., 111(22):10371-10374, (1999).

[15] M. Muthukumar. Phys. Rev. Lett., 86(14):3188-3191, (2001).

[16] C. Dekker. Nature Nanotechnol., 2(4):209-215, (2007).

[17] D. Branton, D. W. Deamer, A. Marziali, H. Bayley, S. A. Benner, T. Butler, M. Di Ventra, S. Garaj, A. Hibbs, X. Huang, S. B. Jovanovich, P. S. Krstic, S. Lindsay, X. S. Ling, C. H. Mastrangelo, A. Meller, J. S. Oliver, Y. V. Pershin, J. M. Ramsey, R. Riehn, G. V. Soni, V. Tabard-Cossa, M. Wanunu, M. Wiggin, and J. A. Schloss. Nature Biotechnol., 26(10):1146-1153, (2008). 
[18] R. Smeets, U. Keyser, D. Krapf, M. Wu, N. Dekker, and C. Dekker. Nano Lett., 6(1):89-95, (2006).

[19] S. van Dorp, U. F. Keyser, N. H. Dekker, C. Dekker, and S. G. Lemay. Nature Phys., 5(5):347-351, (2009).

[20] H. Gao, Y. Kong, D. Cui, and C. Ozkan. Nano Lett., 3(4):471-473, (2003).

[21] R. Fan, R. Karnik, M. Yue, D. Li, A. Majumdar, and P. Yang. Nano Lett., 5(9):1633-1637, (2005).

[22] Y. Xie, Y. Kong, A. K. Soh, and H. Gao. J. Chem. Phys., 127(22):225101, (2007).

[23] Q. X. Pei, C. G. Lim, Y. Cheng, and H. Gao. J. Chem. Phys., 129(12):125101, (2008).

[24] M. C. G. Lim, Q. Pei, and Z. W. Zhong. Physica A, 387(13):3111-3120, (2008).

[25] H. Liu, J. He, J. Tang, H. Liu, P. Pang, D. Cao, P. Krstic, S. Joseph, S. Lindsay, and C. Nuckolls. Science, 327(5961):64-67, (2010).

[26] S. Garaj, W. Hubbard, A. Reina, J. Kong, D. Branton, and J. A. Golovchenko. Nature, 467(7312):190193, (2010).

[27] G. F. Schneider, S. W. Kowalczyk, V. E. Calado, G. Pandraud, H. W. Zandbergen, L. M. K. Vandersypen, and C. Dekker. Nano Lett., 10(8):3163-3167, (2010).

[28] C. A. Merchant, K. Healy, M. Wanunu, V. Ray, N. Peterman, J. Bartel, M. D. Fischbein, K. Venta, Z. Luo, A. T. C. Johnson, and M. Drndic. Nano Lett., 10(8):2915-2921, (2010).

[29] B. M. Venkatesan and R. Bashir. Nature Nanotechnol., 6(10):615-624, (2011).

[30] G. Torrie and J. Valleau. J. Comput. Phys., 23(2):187-199, (1977).

[31] B. Roux. Comput. Phys. Commun., 91(1-3):275-282, (1995).

[32] D. Frenkel, and B. Smit. 2 ed.; Academic Press, (2001).

[33] Y. Duan, C. Wu, S. Chowdhury, M. Lee, G. Xiong, W. Zhang, R. Yang, P. Cieplak, R. Luo, T. Lee, J. Caldwell, J. Wang, and P. Kollman. J. Comput. Chem., 24(16):1999-2012, (2003).

[34] P. K. Maiti, T. A. Pascal, N. Vaidehi, and W. A. Goddard, III. Nuc. Acids Res., 32(20):6047-6056, (2004).

[35] P. K. Maiti, T. A. Pascal, N. Vaidehi, J. Heo, and W. A. Goddard, III. Biophys. J., 90(5):1463-1479, (2006).

[36] T. Darden, D. York, and L. Pedersen. J. Chem. Phys., 98(12):10089-10092, (1993).

[37] I. Yildirim, H. A. Stern, S. D. Kennedy, J. D. Tubbs, and D. H. Turner. J. Chem. Theory Comput., 6(5):1520-1531, (2010).

[38] S.-T. Lin, M. Blanco, and W. A. Goddard, III. J. Chem. Phys., 119(22):11792-11805, (2003).

[39] S.-T. Lin, P. K. Maiti, and W. A. Goddard, III. J. Phys. Chem. B, 114(24):8191-8198, (2010). 
[40] T. A. Pascal, W. A. Goddard, III, and Y. Jung. Proc. Natl. Acad. Sci. USA., 108(29):11794-11798, (2011).

[41] W. C. Still, A. Tempczyk, R. C. Hawley, and T. Hendrickson. J. Am. Chem. Soc., 112(16):6127-6129, (1990).

[42] J. Srinivasan, M. W. Trevathan, P. Beroza, and D. A. Case. Theor. Chem. Acc., 101(6):426-434, (1999).

[43] P. K. Maiti, and B. Bagchi. Nano Lett., 6:2478-2485, (2006).

[44] H. Kumar, B. Mukherjee, S.-T. Lin, C. Dasgupta, A. K. Sood, and P. K. Maiti. J. Chem. Phys., 134(12):124105, (2011).

[45] B. Nandy, and P. K. Maiti. J. Phys. Chem. B, 115:217-230, (2011).

[46] See Supplementary Material Document No. — for the details of non-equilibrium versus equilibrium free energy methods and for more snapshots.

[47] Sponer, J.; Jurecka, P.; Hobza, P. J. Am. Chem. Soc., 126:10142-10151, (2004).

[48] Y. Huang, X. Weng, and I. M. Russu. Biochemistry, 50(11):1857-1863, (2011).

[49] T. Ito, L. Sun, and R. Crooks. Chem. Commun., (13):1482-1483, (2003).

[50] B. Nandy, M. Santosh, and P. K. Maiti. J. Biosci., 37(3):457-474, (2012).

[51] D. Voet and J. G. Voet. John Wiley \& Sons. Inc.,, 3 edition, (2005).

[52] M. Santosh and P. K. Maiti. J. Phys.: Condens. Matter, 21(3):034113, (2009).

[53] M. Santosh and P. K. Maiti. Biophys. J., 101(6):1393-1402, (2011).

[54] R. Hendrix. Proc. Natl. Acad. Sci. USA., 75(10):4779-4783, (1978).

[55] J. Nummela and I. Andricioaei. Biophys. J., 96(4):L29-L31, (2009).

[56] T. Hugel, J. Michaelis, C. L. Hetherington, P. J. Jardine, S. Grimes, J. M. Walter, W. Faik, D. L. Anderson, and C. Bustamante. PLoS Biol, 5(3):558-567, (2007).

[57] W. Humphrey, A. Dalke, and K. Schulten. J. Mol. Graph., 14(1):33-\&, (1996).

\section{Figures}




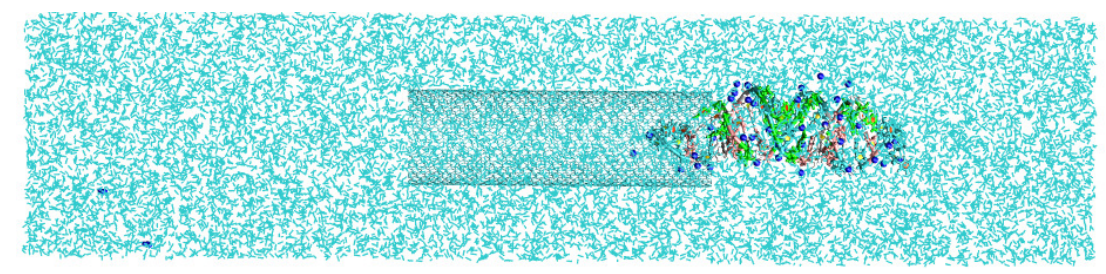

(a)System after equilibration with water and counterions.
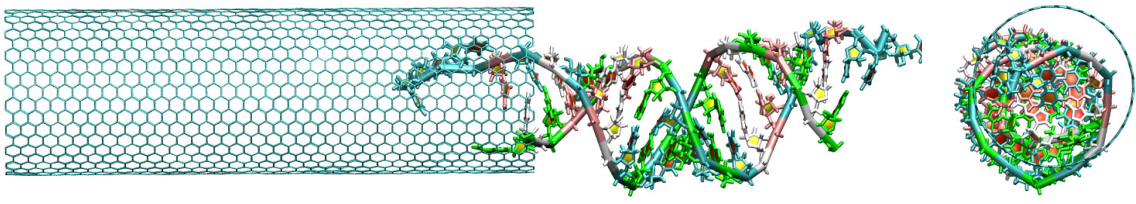

(b) $3 \mathrm{~ns}$

(c)
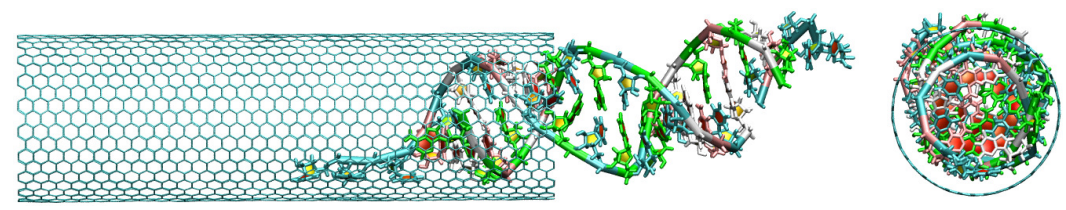

(d) $10 \mathrm{~ns}$

(e)

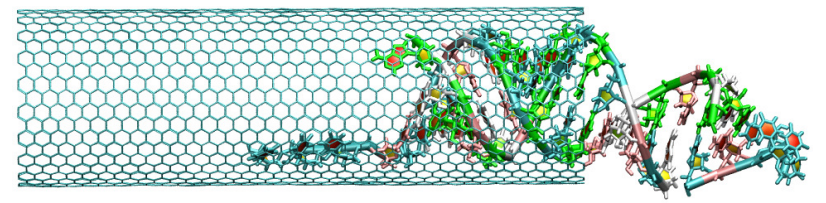

(f) $20 \mathrm{~ns}$

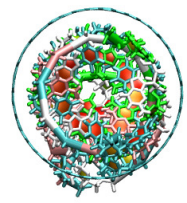

$(\mathrm{g})$

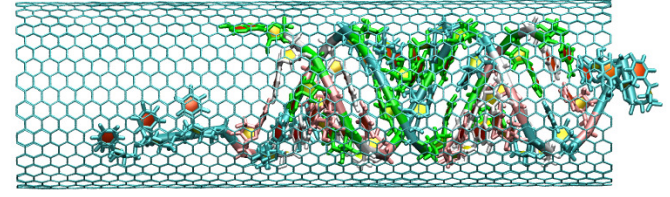

(h) $35 \mathrm{~ns}$

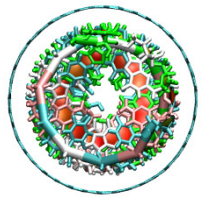

(i)

FIG. 1: (a) The initial system setup for simulation where siRNA-CNT hybrid was solvated with water and neutralizing $\mathrm{Na}^{+}$counterions. (b)-(i) Snapshots of siRNA inside $(20,20) \mathrm{CNT}$ at $3 \mathrm{~ns}, 10 \mathrm{~ns}, 20 \mathrm{~ns}$ and 35 ns during MD simulation in horizontal and vertical view with respect to CNT long axis $\hat{n}$. Water and counterions were not shown in Figures (b)-(i) for clarity. The snapshots in this paper were rendered using VMD software [57]. 


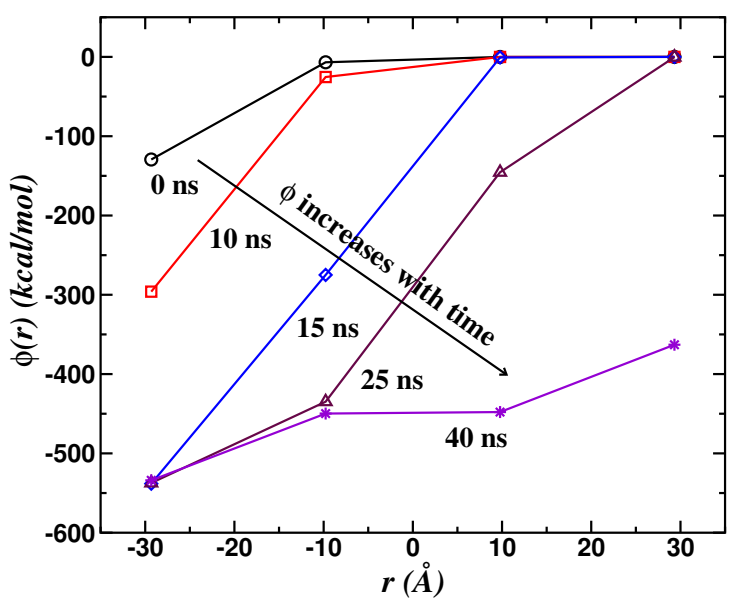

FIG. 2: The van der Waals interaction energy $\phi(r)$ of siRNA that is arising due to the presence of CNT in the system. At $t=0 \mathrm{~ns}$, siRNA is outside CNT with two sticky-ends lying near to one end of the CNT. Hence at $t=0 \mathrm{~ns}$, the interaction of siRNA with CNT is very less. As time progress these sticky-ends would interact with CNT strongly as shown at $t=10,15,25$ and $40 \mathrm{~ns}$.

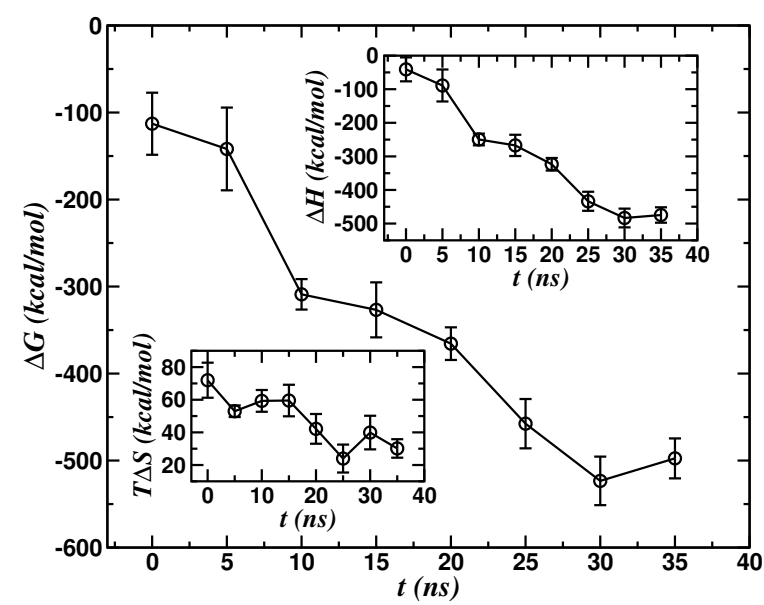

(a)

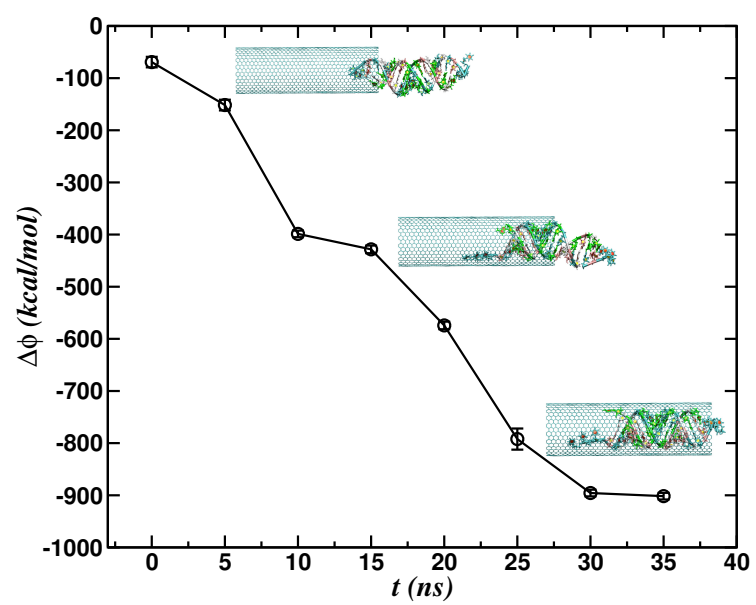

(b)

FIG. 3: (a) Binding free energy as a function of time with entropy and enthalpy shown in insets that are calculated using 2PT and MM-GBSA method, respectively. (b) vdW contribution to $\Delta G$ as a function of time. $\Delta G$ and $\phi$ are increasing with time as siRNA translocates inside $(20,20) \mathrm{CNT}$ and reaches constant after complete translocation. 


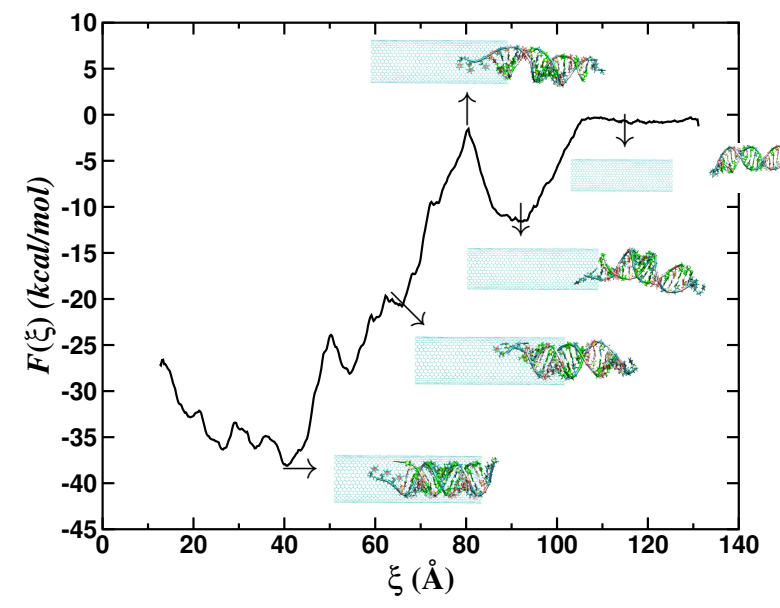

(a)

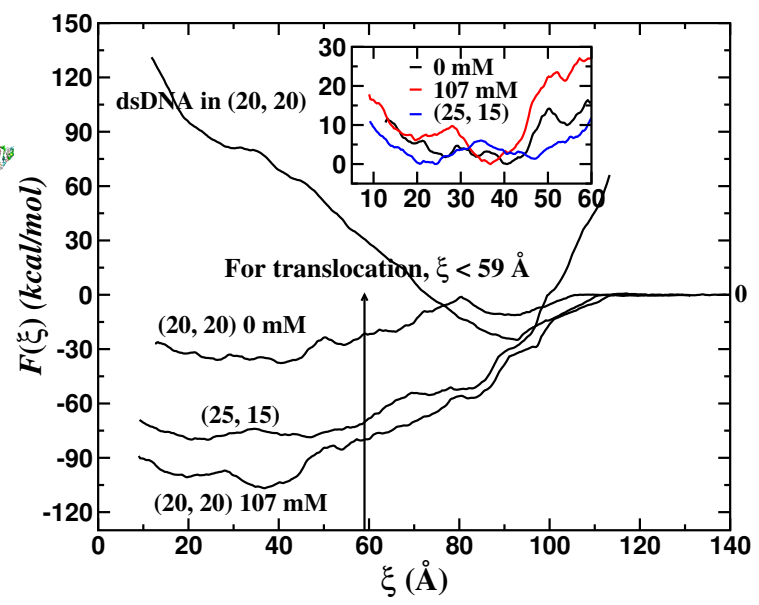

(b)

FIG. 4: (a) Potential of the mean force $F(\xi)$ of siRNA translocation inside $(20,20)$ CNT along the reaction coordinate $\xi$. (b) $F(\xi)$ for siRNA translocation inside $(20,20) \mathrm{CNT}$ at 0 and $107 \mathrm{mM} \mathrm{NaCl},(25,15) \mathrm{CNT}$ and dsDNA translocation inside $(20,20)$ CNT. We define that the siRNA/dsDNA is translocated inside CNT when at least half of the base-pairs of siRNA/dsDNA are inside CNT from which it follows that $\xi \leq 59 \AA$. The inset shows the minima of shifted $F(\xi)$ which is inside CNT for siRNA and outside for dsDNA since it does not translocate. 


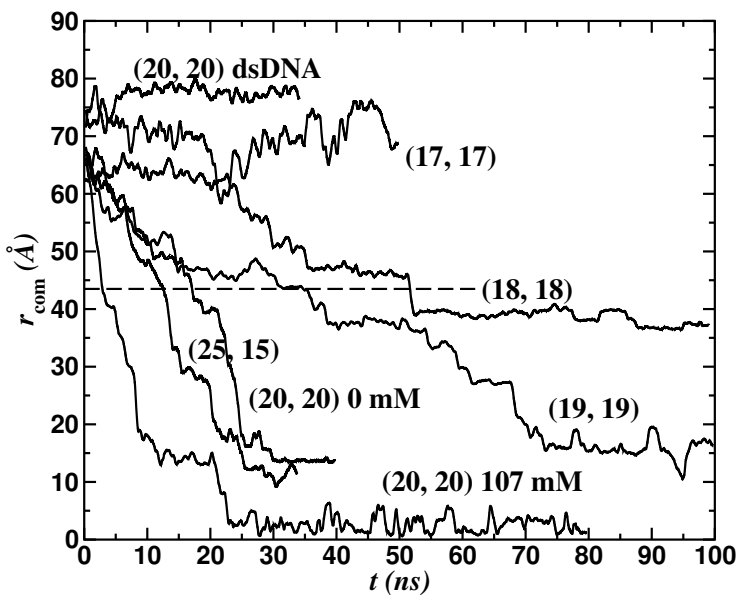

(a)

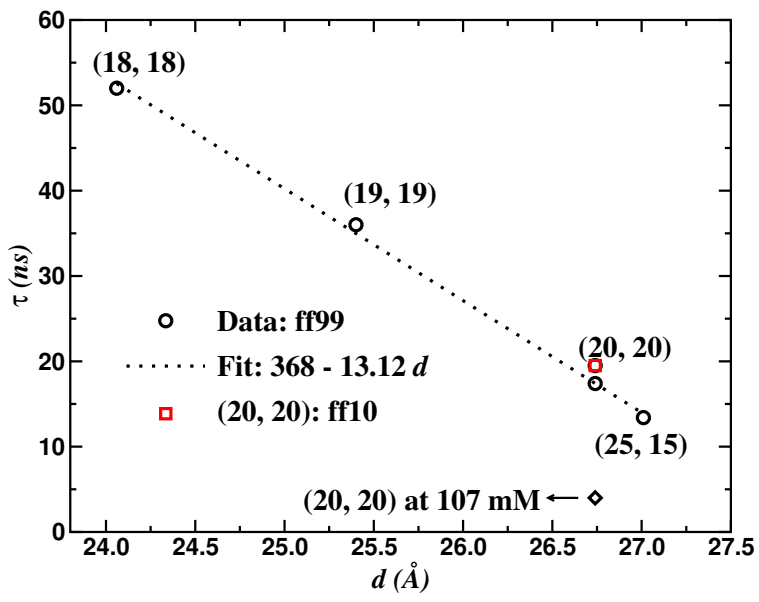

(b)

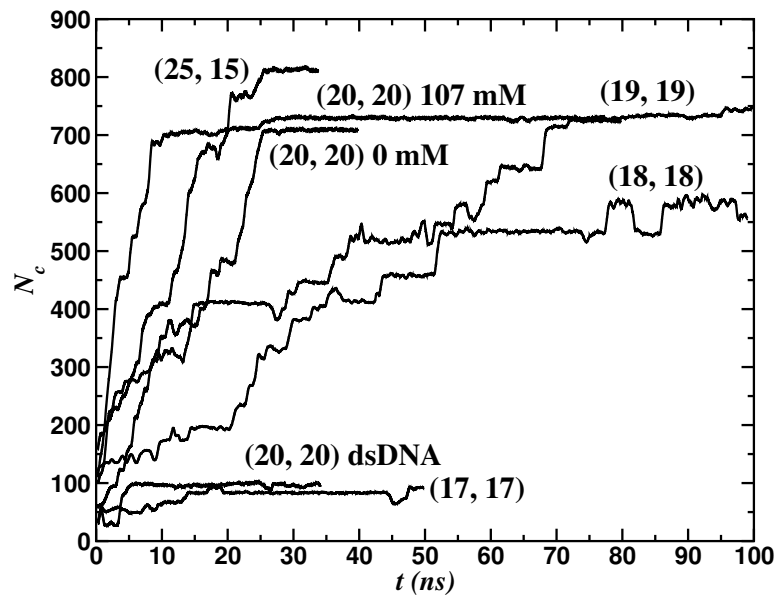

(c)

FIG. 5: (a) The COM distance between siRNA and CNT $\left(r_{\text {com }}\right)$ as shown in inset of supplementary Figure 1(a) for siRNA inside CNT of various diameter and chirality. For the translocation, $r_{\text {com }} \leq L / 2$ implies $r_{\text {com }} \leq 43.5 \AA$ which is marked as horizontal dashed line. (b) Translocation time ( $\tau$ ) of siRNA as a function of the diameter $d$ of CNT using ff99 force field. $\tau$ decreases linearly with increasing $d$. In the Figure, we have also shown $\tau$ for siRNA in $(20,20)$ CNT using ff10 force field as red square mark and siRNA in (20, 20) $\mathrm{CNT}$ at $107 \mathrm{mM}$ as diamond mark. (c) Number of close contacts $\left(N_{c}\right)$ 


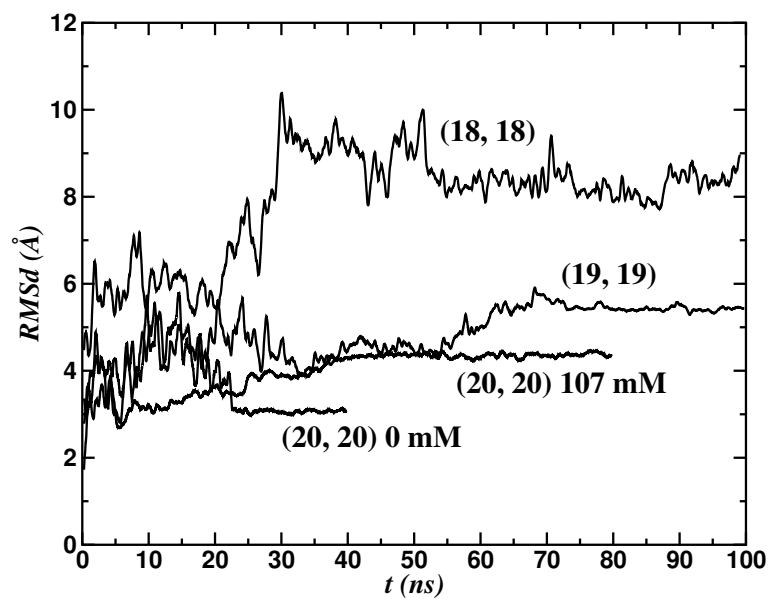

(a)

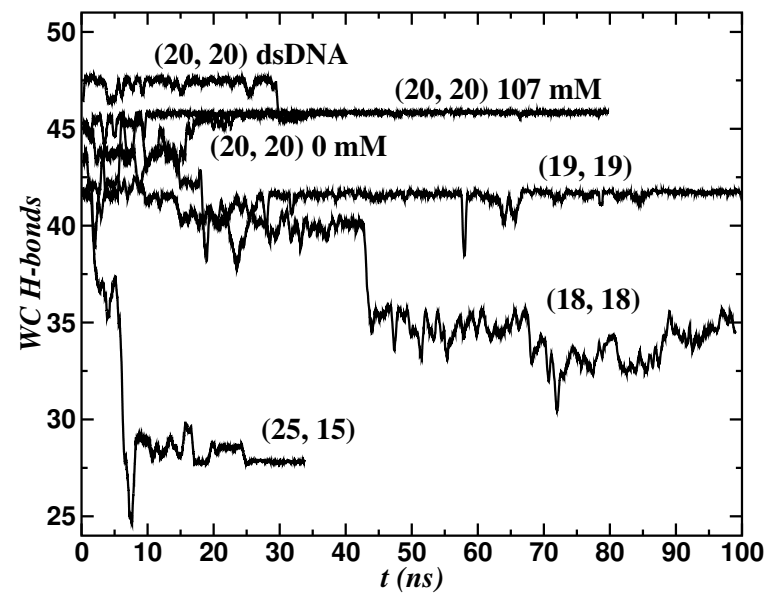

(b)

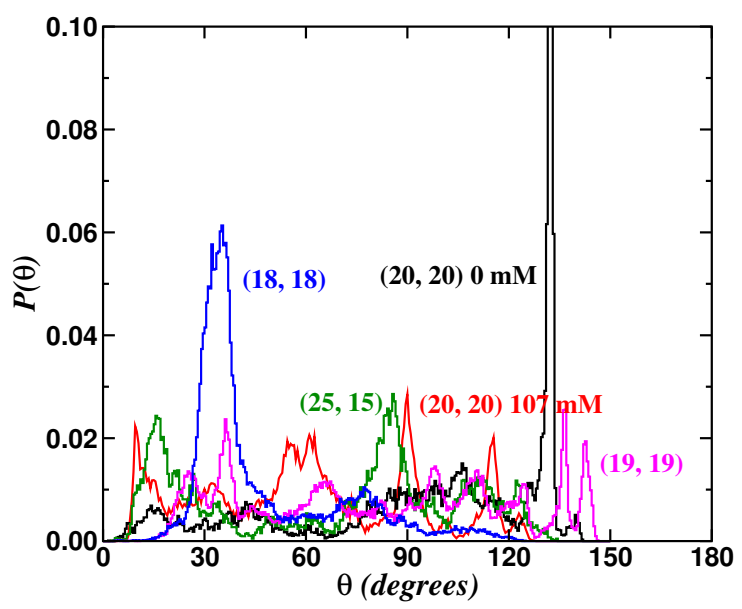

(c)

FIG. 6: Structural deformation: (a) RMSd and (b) WC H-bonds of siRNA as a function of time. These structural parameters are fluctuating before complete translocation and reaches stable constant value after the complete translocation of siRNA. This also indicates that siRNA-CNT hybrid is stable after translocation. (c) Probability of rotation angle, $\theta$ of siRNA.

\section{Tables}

TABLE I: Summary of the simulation setup

\begin{tabular}{|l|l|l|l|l|l|l|l|}
\hline$(\mathrm{m}, \mathrm{n}) \mathrm{CNT}$ & siRNA & Box Dimensions $\left(\AA^{3}\right)$ & $\mathrm{Na}^{+}$ & $\mathrm{Cl}^{-}$ & $c(m M)$ & WAT & Total Atoms \\
\hline
\end{tabular}




\begin{tabular}{|l|c|c|c|c|c|c|c|}
\hline$(20,20) 2880$ & 1396 & $74 \times 74 \times 320$ & 44 & 0 & $0[\mathrm{NaCl}]$ & 47619 & 147177 \\
$(20,20) 2880$ & 1396 & $74 \times 74 \times 320$ & 157 & 113 & $107[\mathrm{NaCl}]$ & 47395 & 146731 \\
$(19,19) 2736$ & 1396 & $73 \times 73 \times 318$ & 44 & 0 & $0[\mathrm{NaCl}]$ & 45897 & 141867 \\
$(18,18) 2592$ & 1396 & $72 \times 72 \times 319$ & 44 & 0 & $0[\mathrm{NaCl}]$ & 44393 & 137211 \\
$(17,17) 2448$ & 1396 & $70 \times 70 \times 320$ & 44 & 0 & $0[\mathrm{NaCl}]$ & 42690 & 131958 \\
$(25,15) 2940$ & 1396 & $74 \times 74 \times 318$ & 44 & 0 & $0[\mathrm{NaCl}]$ & 47358 & 146454 \\
$(20,20) 2880$ & 1398 & $74 \times 74 \times 321$ & 42 & 0 & 0 dsDNA $[\mathrm{NaCl}]$ & 47849 & 147867 \\
\hline
\end{tabular}




\title{
Translocation and encapsulation of siRNA inside carbon nanotubes
}

\author{
Santosh Mogurampelly* and Prabal K. Maiti ${ }^{\dagger}$ \\ Centre for Condensed Matter Theory, Department of Physics, \\ Indian Institute of Science, Bangalore 560012, India
}

*To whom correspondence should be addressed; Electronic address: santosh@physics.iisc.ernet.in

†Electronic address: maiti@physics.iisc.ernet.in 


\section{NON-EQUILIBRIUM VERSUS EQUILIBRIUM FREE ENERGY METHODS}

Transformation of binding energy from a function of simulation time $t$ to a function of $r_{\text {com }}$, center of mass distance between CNT and siRNA is carried out (See the schematic in Figure 1(a) for the pictorial definition of $\left.r_{\mathrm{com}}\right)$. The transformation is done simply by monitoring $r_{\mathrm{com}}(t)$. For the Umbrella sampling calculations, a new distance parameter $\xi$, called reaction coordinate is introduced. For simplicity in implementation without loss of generality and understanding, we calculate $\xi$ as the distance between the center of masses of 'far end' of CNT and first two WatsonCrick (WC) H-bonded base-pairs of siRNA near to the CNT (See schematic in Figure 1(a)). For the notations to follow, we use the term 'un-biased' for the MD simulation that are performed without any external biased forces and 'biased' for the MD simulations with external bias as the case of Umbrella sampling. In the un-biased MD simulations, the corresponding parameter for $\xi$ is $\xi_{\text {un-biased. We plot }} \xi_{\text {un-biased }}$ as a function of $r_{\text {com }}(t)$ in Figure 1(a). Due to the effect of Umbrella biasing potential there could be minor structural deformations in siRNA leading to different values of $\xi$ and $\xi_{\text {un-biased }}$ for same value of $r_{\text {com }}$ in both the biased and un-biased simulations. Using the $\xi$ values from Umbrella sampling, we plot $\xi$ as a function of $\xi_{\text {un-biased }}$ in the inset of Figure 1(b). By assuming (fitting) $\xi$ to depend linearly on $\xi_{\text {un-biased }}$; i.e., $\xi=m \xi_{\text {un-biased }}+c$, we arrive at $m=1.2022$ and $c=-13.456$. This calibration procedure helps us to calculate $\Delta G$ as a function of $\xi$ as shown in Figure 1(b) which is more relevant to compare with Umbrella sampling results. As can be seen in Figure 1(b), $\Delta G$ seems to have a global minima at $\xi=22 \AA$ where siRNA is inside $(20,20) \mathrm{CNT}$ as the inset snapshot indicates. At $\xi=78 \AA$ or more, siRNA is away from CNT which is at peak of unstable configuration. Intermediate values of $\xi$ are also shown with snapshots. The limitation in calculating $\Delta G$ as a function of $\xi$ described above is that the system is not well sampled at each

$\xi$ to have an ensemble averages since the translocation process is spontaneous. To improve the sampling at each $\xi$ to great level and obtain $\Delta G$ to great accuracy, we employ Umbrella sampling method as described in the previous section. Snapshots of siRNA in $(20,20)$ CNT are shown in Figure 2 at $40 \mathrm{~ns}$ in perspective display mode. 
[1] G. Torrie, and J. Valleau. J. Comput. Phys., 23, 187-199, (1977)

[2] B. Roux. Comput. Phys. Commun., 91(1-3):275-282, (1995).

[3] D. Frenkel, and B. Smit. 2 ed.; Academic Press, (2001)

\section{Figures}

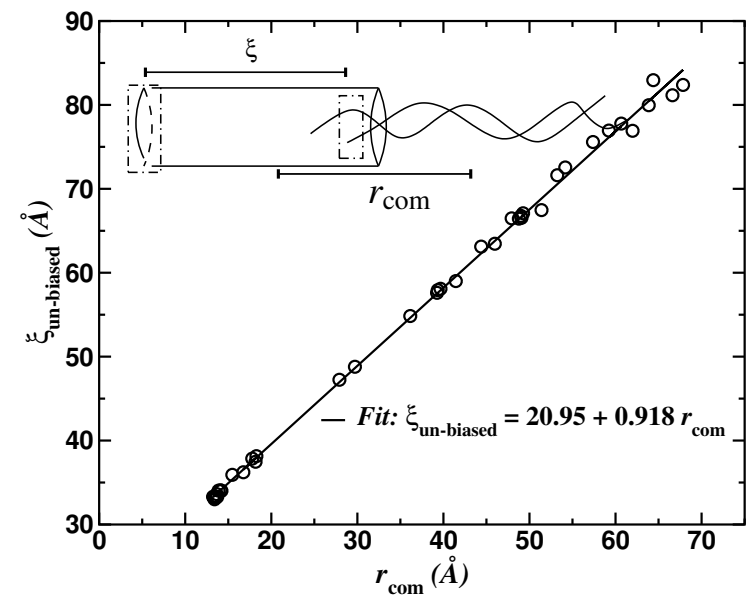

(a)

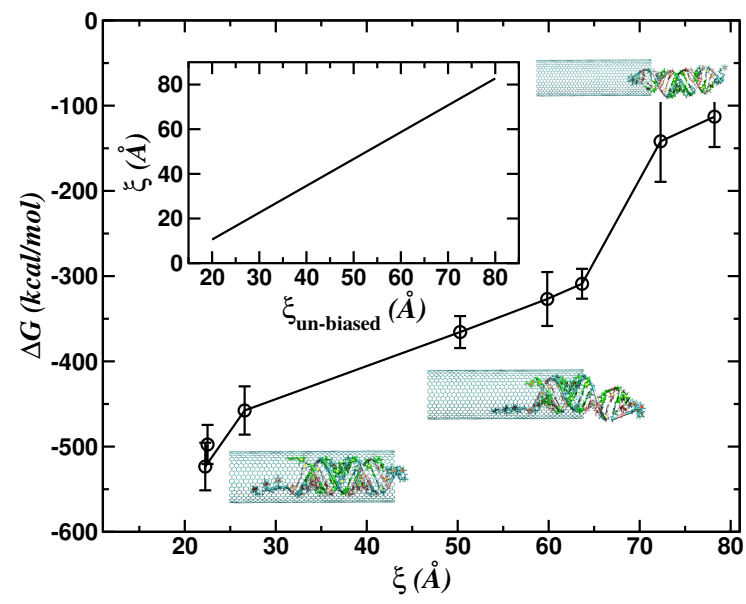

(b)

FIG. 1: (a) Dependency of $\xi_{\text {un-biased }}$ on $r_{\text {com }}$ from which $\xi$ can be calculated as $\xi=1.2 \xi_{\text {un-biased }}-13.456$ as shown in inset of Figure 1(b); where $\xi$ is the distance between the center of masses of 'far end' of CNT and first two Watson-Crick (WC) H-bonded base-pairs of siRNA near to the CNT as shown in inset cartoon, $\xi_{\text {un-biased }}$ is the corresponding parameter in un-biased simulations. (b) $\Delta G$ as a function of reaction coordinate $\xi$ as defined in the Umbrella sampling for comparison. 


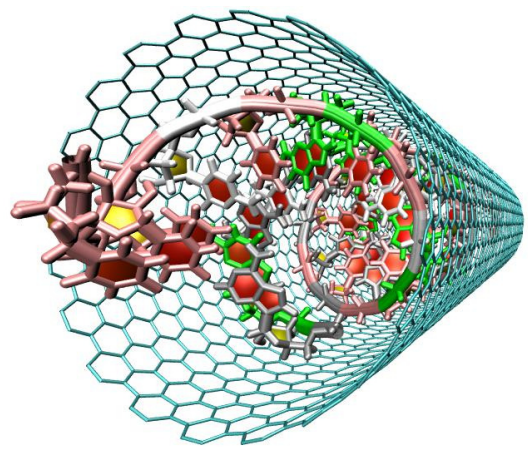

(a)

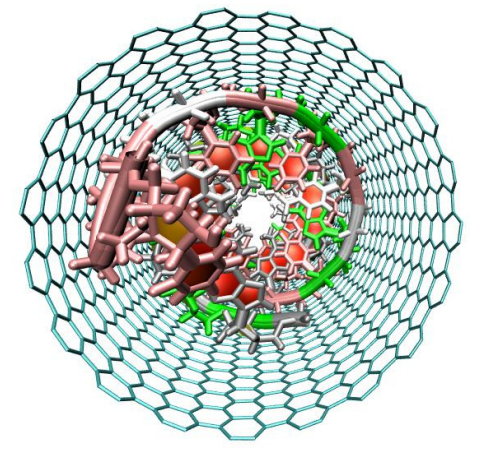

(b)

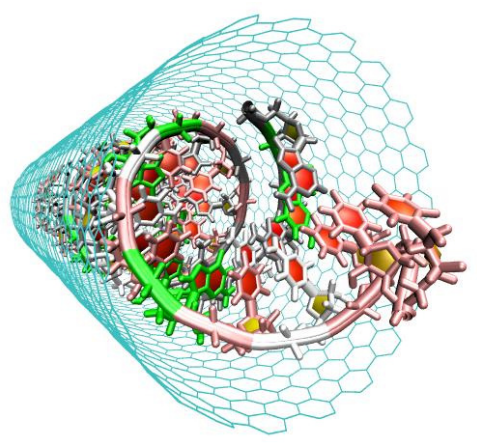

(c)

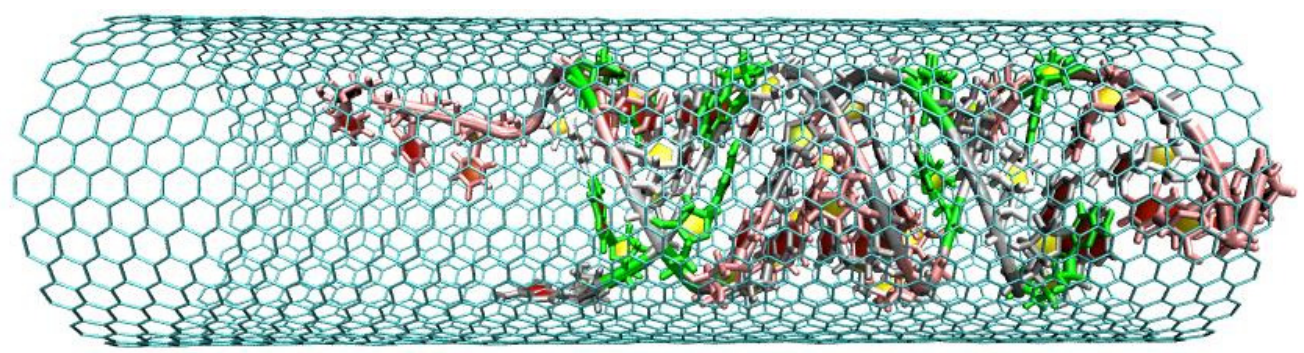

(d)

FIG. 2: Snapshots of siRNA after complete translocation inside $(20,20)$ CNT. These snapshots were rendered at $39 \mathrm{~ns}$ in perspective display mode. 